\title{
Phenomenological bound on the viscosity of the hadron resonance gas
}

\author{
Snigdha Ghosh, ${ }^{1,2, *}$ Sabyasachi Ghosh, ${ }^{3, \dagger}$ and Sumana Bhattacharyya ${ }^{4, \ddagger}$ \\ ${ }^{1}$ Indian Institute of Technology Gandhinagar, Palaj, Gandhinagar 382355, Gujarat, India \\ ${ }^{2}$ Variable Energy Cyclotron Centre, 1/AF Bidhannagar, Kolkata 700064, India \\ ${ }^{3}$ Indian Institute of Technology Bhilai, GEC Campus, Sejbahar, Raipur 492015, Chhattisgarh, India \\ ${ }^{4}$ Center for Astroparticle Physics and Space Science, Bose Institute, Block EN, Sector V, Salt Lake, Kolkata 700091, India
}

(Received 6 December 2017; revised manuscript received 9 August 2018; published 8 October 2018)

\begin{abstract}
We explore some phenomenological issues during calculations of transport coefficients for hadronic matter, produced in the experiments of heavy ion collisions. Here, we use an ideal hadron resonance gas model to demonstrate the issues. On the basis of dissipation mechanism, the hadronic zoo is classified into resonance and nonresonance members who participate in dissipation via strong decay and scattering channels, respectively. Imposing our phenomenological restriction, we are able to provide a rough upper and lower bound estimations of transport coefficients. Interestingly, we find that our proposed lower limit estimation for shear viscosity to entropy density ratio is a little larger than its quantum lower bound. By taking a simple example, we demonstrate how our proposed restriction helps to tune any estimation of transport coefficients within its numerical band, proposed by us.
\end{abstract}

DOI: 10.1103/PhysRevC.98.045202

\section{INTRODUCTION}

The shear viscosity $(\eta)$ to entropy density $(s)$ ratio is the measure of the fluidity of the medium. Being roughly proportional to the ratio of the mean-free path to the de Broglie wavelength of medium constituent, the $\eta / s$ of any fluid can never be vanished because the mean-free path of any constituent can never be lower than its de Broglie wavelength. It indicates that quantum fluctuations prevent the existence of a perfect fluid in nature and the $\eta / s$ of any fluid should have some lower bound, which is also claimed from the string theory calculation [1]. Interestingly, a small value of $\eta / s$, close to this quantum lower bound, is observed in a superhot medium, produced in the relativistic heavy ion collision experiment as well as in some other many-body systems like cold atoms [2], graphene [3], and in low-energy nuclear matter [4]. This nearly perfect fluid behavior, at extreme conditions, has drawn immense attention from scientific communities working on the field of condensed matter physics to nuclear physics to string theory.

In our present work, we emphasize some phenomenological issues of $\eta / s$ for hadronic matter. We have a long list of Refs. [5-25], which addressed different microscopic calculations of this $\eta / s$, based on different hadronic models [5-14], different effective QCD models [15-21], and bulk

\footnotetext{
*snigdha.physics@gmail.com

†'sabyaphy@gmail.com

${ }^{\ddagger}$ response2sumana91@gmail.com
}

Published by the American Physical Society under the terms of the Creative Commons Attribution 4.0 International license. Further distribution of this work must maintain attribution to the author(s) and the published article's title, journal citation, and DOI. Funded by $S C O A P^{3}$. simulations [22-26]. The predicted values of $\eta / s$ from earlier estimations reside within a broad numerical band. The same is observed for bulk viscosity $\zeta[5,8,13-20,27-44]$.

Here we find a possibility of comparatively narrower band for the $\eta / s$ of hadronic matter when we put a restriction in the calculations of $\eta / s$ based on an ideal hadron resonance gas (HRG) model. The restriction is to consider the dissipation of hadrons within a finite size of matter produced at the BNL Relativistic Heavy Ion Collider (RHIC) (similar for CERN Large Hadron Collider) or Large Hadron Collider (LHC). When we follow the expressions of different transport coefficients in the framework of relaxation time approximation (RTA), we assume that the relaxation length (time) should be lower than the size (life time) of the system or medium. Owing to this fact, when we consider the hadronic matter, the resonances, whose mean life time are larger than the life time of the system, will not take part in the dissipation process. So we have to eliminate them during the calculation of transport coefficients for finite-size hadronic matter. On the other hand, the hadrons like pion, kaon, and nucleon can have a momentum-dependent relaxation length, whose high momentum component may become larger than the system size. Therefore, we have to eliminate the high-momentum part by imposing an upper momentum cutoff in the calculation. This fact of finite-size dissipation is pointed out in the present work with the help of the ideal HRG model. A generic qualitative message of the present study is that the theoretical tools should have to take care of this fact of finite-size dissipation when we try to give the estimation of transport coefficients for RHIC or LHC matter.

The article is organized as follows. Next, in the formalism part (Sec. II), first (Sec. II A) we address the standard expression of different transport coefficients and then (Sec. II B) we provide a brief description of ideal HRG model, 
whose detail expressions are given in the Appendix. After getting the expression of transport coefficients and thermodynamical quantity like entropy density, they are folded by the spectral function of hadrons. Its generic equation is written in the subsection (Sec. II C) of the formalism part. Next we will come to the results section (Sec. III), where we explore the issues of finite-size dissipation, which can give us a rough numerical band in the values of different transport coefficients. Then, we provide an example of microscopic calculation of transport coefficients, whose values do not remain within our proposed band but after utilizing the appropriate restriction of finite-size dissipation, we get their modified values, which ultimately remain within our proposed band. At last, we summarize our studies in Sec. IV.

\section{FORMALISM}

\section{A. Transport coefficients in Kubo formalism}

Our aim of this work is to calculate these transport coefficients with the help of HRG model, so we have to add the contributions of all mesons $(M)$ and baryons $(B)$ to obtain total transport coefficients of hadronic matter. We know that the mathematical structure of transport coefficients, obtained from the one-loop diagram in the quasiparticle Kubo approach and relaxation time approximation (RTA) in the kinetic theory approach, are exactly same. Without going into those background formalism parts of transport coefficients like shear viscosity $\eta[8,9,44,45]$ and bulk viscosity $\zeta[8,18]$, let us start with their standard expressions

$$
\begin{aligned}
\eta= & \sum_{h \in\{\text { hadrons }\}} \frac{g_{h}}{15 T} \int \frac{d^{3} \vec{k}}{(2 \pi)^{3}} \tau_{h}\left(\frac{\vec{k}^{2}}{\omega_{h}}\right)^{2} f_{h}\left(1-a_{h} f_{h}\right), \\
\zeta= & \sum_{h \in \text { hadrons }\}} \frac{g_{h}}{T} \int \frac{d^{3} \vec{k}}{(2 \pi)^{3} \omega_{h}^{2}} \tau_{h}\left\{\left(\frac{1}{3}-c_{s}^{2}\right) \vec{k}^{2}-c_{s}^{2} m_{h}^{2}\right\}^{2} \\
& \times f_{h}\left(1-a_{h} f_{h}\right),
\end{aligned}
$$

where $g_{h}, \omega_{h}=\sqrt{\vec{k}^{2}+m_{h}^{2}}$, and $f_{h}=\left[e^{\omega_{h} / T}+a_{h}\right]^{-1}$ are, respectively, the degeneracy factor, energy, and thermal distribution function (Fermi-Dirac or Bose-Einstein) of hadron $h$; $a_{h}=+1$ if $h$ is a fermion and $a_{h}=-1$ if $h$ is a boson. In the above equation, $\tau_{h}$ is the relaxation time of $h$, which proportionally controls the numerical strength of the transport coefficients. Obviously, the thermal phase-space factors, depend on the thermal distribution functions of different hadrons, are another controlling component for transport coefficients.

\section{B. Thermodynamics from ideal HRG}

As we are interested in the (nearly) perfect fluid nature of the medium, produced in HIC experiments, so we focus on the quantity-fluidity, which is quantified by the $\eta / s$, where $S$ is the entropy density. To calculate the $s$ of hadronic matter, we follow the standard procedure of the ideal HRG model [46], where all thermodynamic quantities like energy density $(\epsilon)$, pressure $(P)$, entropy density $(s)$, speed of sound $\left(c_{s}\right)$, and so on are calculated from the partition function. The Grand
Canonical partition function is given by

$$
\begin{aligned}
\ln \mathcal{Z}(T, V,\{\mu\}) \\
=V \int \frac{d^{3} p}{(2 \pi)^{3}} \sum_{h \in\{\text { hadrons }\}} g_{h} a_{h} \\
\ln \left[1+a_{h} \exp \left\{-\beta\left(\omega_{h}-\sum_{\mu_{k} \in\{\mu\}} q_{h}^{k} \mu_{k}\right)\right\}\right],
\end{aligned}
$$

where $\{\mu\}=\left\{\mu_{B}, \mu_{Q}, \mu_{S}, \ldots,\right\}$ is the set of chemical potentials corresponding to the conserved quantities [like net baryon $\left(n_{B}\right)$, net charge $\left(n_{Q}\right)$, net strangeness $\left(n_{S}\right)$ etc.] and $q_{h}^{k}$ is the corresponding quantum number of the $h$ th hadron. From the partition function, all the thermodynamic quantities can be calculated as follows:

$$
\begin{aligned}
P & =\left(\frac{T}{V}\right) \ln \mathcal{Z} \\
\varepsilon & =\left(\frac{T^{2}}{V}\right) \frac{\partial}{\partial T}(\ln \mathcal{Z}) \\
n_{k} & =\left(\frac{T}{V}\right) \frac{\partial}{\partial \mu_{k}}(\ln \mathcal{Z}) ; k=B, Q, S, \ldots \\
c_{s}^{2} & =\left(\frac{\partial p}{\partial \epsilon}\right)=\left(\frac{\partial p}{\partial T}\right) /\left(\frac{\partial \epsilon}{\partial T}\right)+\sum_{\mu_{k} \in\{\mu\}}\left(\frac{\partial p}{\partial \mu_{k}}\right) /\left(\frac{\partial \epsilon}{\partial \mu_{k}}\right)
\end{aligned}
$$

The entropy density $s$ can be obtained from

$$
s=\left(\frac{\varepsilon+P}{T}\right)-\frac{1}{T} \sum_{\mu_{k} \in\{\mu\}} n_{k} \mu_{k} .
$$

The momentum integration in Eq. (3) can be analytically performed in terms of modified Bessel function details of which are provided in Appendix. In this work we take $\mu_{B}=$ $\mu_{Q}=\mu_{S}=\cdots=0$, which implies that $\{\mu\}$ is a null set.

\section{Spectral folding}

The transport coefficients as well as the thermodynamic quantities as given in Eqs. (1)-(2), (4)-(8), depend on the masses of all the hadrons $\left\{m_{h}\right\}$. To take into account the finite widths of the unstable hadrons, the transport coefficients and the thermodynamic quantities are folded with the corresponding hadronic spectral functions $\rho_{h}^{\mathrm{m}, \mathrm{b}}(M)$. Let $\Phi$ denotes any of the transport coefficients (such as $\eta, \zeta$ ) or thermodynamic quantities (such as $\varepsilon, P$, $s$, etc.). In this work, the spectral foldings are done through

$$
\begin{aligned}
\Phi_{\text {folded }}= & \sum_{h \in\{\text { mesons }\}} \frac{1}{N_{h}^{\mathrm{m}}} \int_{\left(m_{h}-2 \Gamma_{h}\right)^{2}}^{\left(m_{h}+2 \Gamma_{h}\right)^{2}} d M^{2} \rho_{h}^{\mathrm{m}}(M) \Phi\left(m_{h}=M\right) \\
& +\sum_{h \in\{\text { baryons }\}} \frac{1}{N_{h}^{\mathrm{b}}} \int_{m_{h}-2 \Gamma_{h}}^{m_{h}+2 \Gamma_{h}} d M \rho_{h}^{\mathrm{b}}(M) \Phi\left(m_{h}=M\right),
\end{aligned}
$$



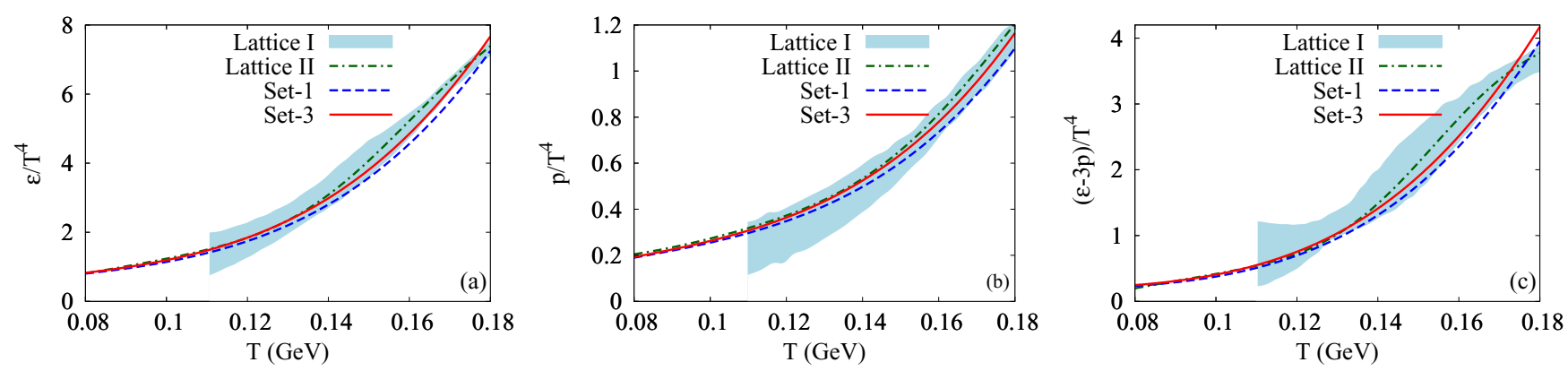

FIG. 1. (a) Energy density, (b) pressure, and (c) trace anomaly scaled with fourth power of inverse temperature as a function of temperature compared among results from ideal HRG and two lattice QCD data from Refs. [47] and [48] abbreviated as Lattice I and Lattice II, respectively. Set-1 and Set-3 (see Table II) correspond to results from ideal HRG without and with spectral folding.

with $\quad N_{h}^{\mathrm{m}}=\int_{\left(m_{h}-2 \Gamma_{h}\right)^{2}}^{\left(m_{h}+2 \Gamma_{h}\right)^{2}} d M^{2} \rho_{\mathrm{m}}(M) \quad$ and $\quad N_{h}^{\mathrm{b}}=\int_{m_{h}-2 \Gamma_{h}}^{m_{h}+2 \Gamma_{h}}$ $d M \rho_{\mathrm{b}}(M)$. In the above equation, the mesonic and baryonic spectral functions are, respectively,

$$
\begin{aligned}
\rho_{h}^{\mathrm{m}}(M) & =\frac{1}{\pi} \operatorname{Im}\left[\frac{1}{M^{2}-m_{h}^{2}+i M \Gamma_{h}}\right], \\
\rho_{h}^{\mathrm{b}}(M) & =\frac{1}{\pi} \operatorname{Im}\left[\frac{1}{M-m_{h}+\frac{i}{2} \Gamma_{h}}\right] .
\end{aligned}
$$

\section{NUMERICAL RESULTS}

Let us begin this section by showing numerical results for the thermodynamic quantities obtained from ideal HRG model in Figs. 1 and 2.

From Eqs. (5) and (4), one can obtain $\epsilon$ and $P$, which are shown by the blue dashed line in Figs. 1(a) and 1(b). Using the folding technique, as given in Eq. (9), the values of $\epsilon$ and $P$ are a little bit enhanced as shown by the red line in Figs. 1(a) and 1(b). Similar kinds of results for trace anomaly $(\epsilon-3 P) / T^{4}$ are shown in Fig. 1(c). One of the success of HRG model is that its estimated values of different thermodynamical quantities are quite close to the results, obtained by lattice quantum chromo dynamics (LQCD). We added two sets of lattice QCD data from Refs. [47] (cyan band) and [48] (green dashed dot line), which are in good agreement with the HRG results of the present work. In the same pattern, the results of $C_{S}^{2}$ and entropy density $s$ are also plotted in Figs. 2(a) and 2(b).

Now let us come to the results of transport coefficients. In the expression of $\eta, \zeta$, given in Eqs. (1) and (2), we see that the thermodynamical phase space parts of different hadrons are known components from the HRG model but their relaxation times are unknown components, which we should have to include in the model from outside, based on our phenomenological understanding. Owing to this phenomenological picture of relaxation of different hadrons, we classified them into two categories-nonresonance (NR) and resonance (R) components. Let us call the pseudoscalar meson nonet and baryon octet as NR members as these long-lived particles cannot decay inside the fireball, produced in HIC experiments. Among them, the pion, kaon, and nucleon are the most abundant constituents in the medium, hence we consider only them as NR members for simplicity. Their strong interaction elastic scattering will provide their relaxation times, which are expected to be important in dissipation within the life time of the fireball. The hadrons, other than the pseudoscalar meson nonet and baryon octet, are considered as $\mathrm{R}$ members as the maximum of them follow strong decays. Their mean life times, which are the inverse of their strong decay widths, are comparable to the life time of the fireball. So these hadrons also live in the fireball along with the NR members but they exist in resonance states. The maximum of them decays within the medium during its life time and therefore their strong
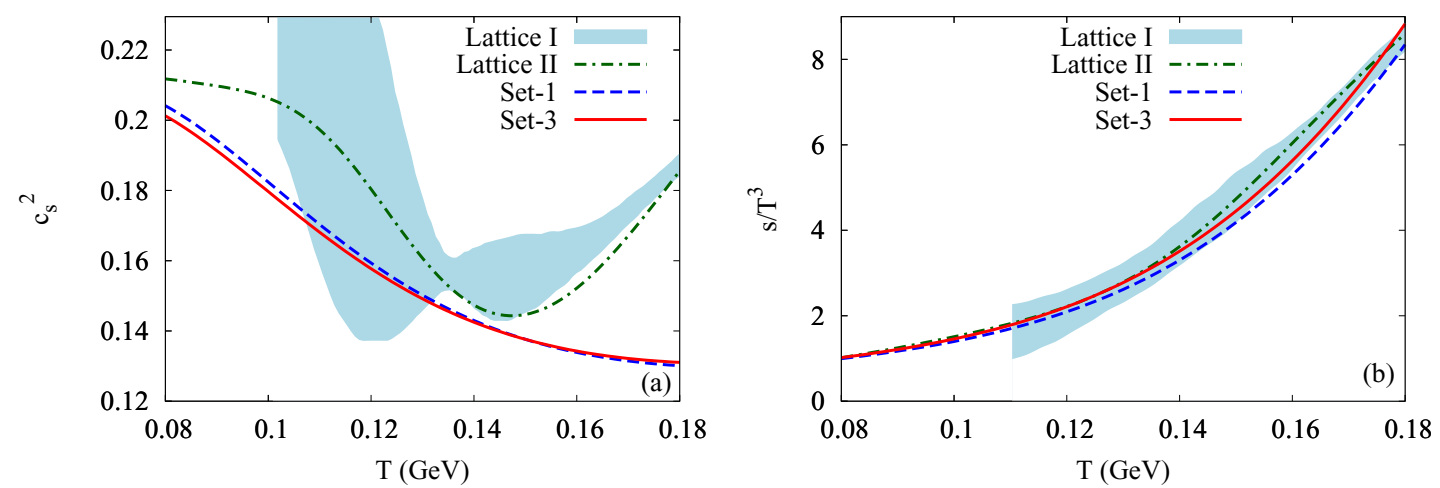

FIG. 2. (a) Speed of sound and (b) entropy density scaled with third power of inverse temperature as a function of temperature compared among results from ideal HRG and two lattice QCD data from Refs. [47] and [48] abbreviated as Lattice I and Lattice II, respectively. Set-1 and Set-3 (see Table II) correspond to results from ideal HRG without and with spectral folding. 


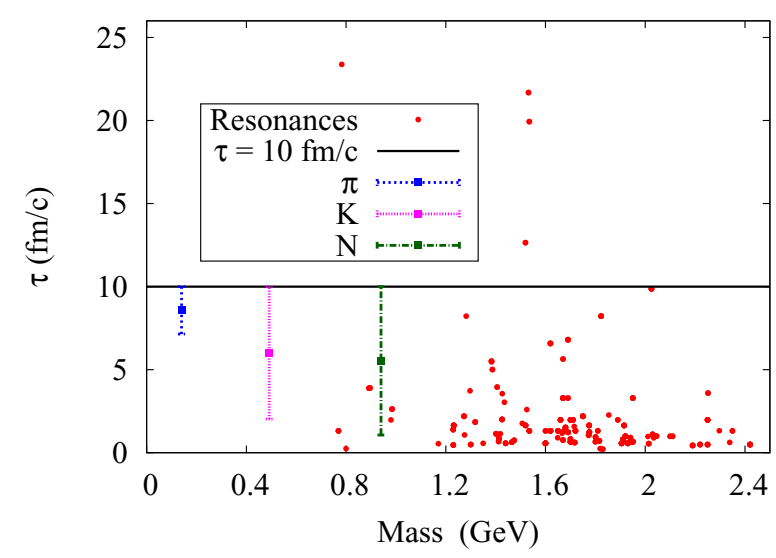

FIG. 3. The values of mean life times (red points) for different hadron resonances up to $2.5 \mathrm{GeV}$ masses. The horizontal line indicates an approximated life time of the hadronic medium, produced in heavy ion experiments. Blue, pink, and green bars denote the ranges of collisional time or relaxation time for nonresonance members $\pi, K$, and $N$, respectively.

decays contribute to the total dissipation of the medium. So, by ignoring other interactions (weak and electromagnetic decays and scattering channels) [15], the strong interaction scale is our matter of interest for calculating dissipation in the medium, which survives in that scale. So we see that $\mathrm{R}$ and NR members participate in dissipation by their strong decay and scattering processes, respectively.

To explore our phenomenological studies on the dissipation process, we plotted the mean life times (red circles) of different hadrons up to $M=2.5 \mathrm{GeV}$ in Fig. 3, which is basically covering the strong interaction spectra of the hadronic zoo at a glance. The horizontal blue dashed line indicates the life time of the fireball, which is approximately taken as $10 \mathrm{fm}$. Hence, the hadrons, whose mean life times are less than the life time of the medium, decay inside the medium and they will only participate in the dissipation. Considering only those hadrons and using their mean life times as the relaxation times $\tau$ in Eq. (1), we will get the shear viscosity of $\mathrm{R}$ component. One has to always consider this amount of shear viscosity for hadronic matter, which may be considered as a lower estimation of $\eta$ in HRG even when we do not take any NR contribution. Now, we focus on the NR component, whose in-medium scattering contribution will not be a fixed value like the $\mathrm{R}$ component. In different hadronic model calculations [5-11], we notice different numerical strengths of $\eta$ from this NR component, although some Refs. [7-10] are concentrated only in pion medium. Let us take the Compton lengths $\left(1 / m_{\pi, K, N}\right)$ of NR particles $(\pi, K, N)$ as their minimum scattering lengths in Eq. (1) and then add this contribution with the $\mathrm{R}$ component to get an approximate lowest estimation of total $\eta$. On the other hand, the life time (maximum size) of the fireball can be considered as the upper limit of relaxation times (relaxation lengths) of NR particles and after adding this contribution with the $R$ part contribution, we get an upper limit estimation of $\eta$. These ranges of relaxation times for $\pi, K$, and $N$ are shown by the blue, pink, and green bars in Fig. 3 and using these ranges, we get a numerical band of $\eta$, which is shown by cayan color in Fig. 4(a).

Normalizing this $\eta$ by that entropy density $s$, we get a similar numerical band for the $\eta / s$ ratio, as shown in Fig. 4(b). In Fig. 4(b), we show that the lowest possible value of $\eta / s$ is a little greater than its quantum lower bound $\left(\frac{\eta}{s}=\frac{1}{4 \pi} \approx 0.08\right)$. At $T=0.160 \mathrm{GeV}$, our proposed band provides an approximate inequality $0.3<\frac{\eta}{s}<0.85$. Now, analyzing the earlier estimations of $\eta / s$ for hadronic matter $[6,8,9,11-14,22,25]$, we see that $\eta / s(T=0.160 \mathrm{GeV}) \approx 0.8$ [6], 0.45 [12], 0.32 [9], $0.3[8,14]$ remain within the inequality, except $\eta / s(T=$ $0.160 \mathrm{GeV}) \approx 1$ [22,25], 0.2 [13], and 0.13 [11]. Whereas at freeze-out temperature $T=0.100 \mathrm{GeV}$ (say), their $\eta / s$ $(\approx 2$ [12], 1.2 [6], 1 [13,22,25], 0.9 [8,9], 0.45 [11], 0.4 [14]) are not at all located within our proposed inequality $0.007<$ $\eta / s<0.4$. The absence of $\mathrm{R}$ members in some formalism $[6,8,9,11]$ and the absence of considering dissipation of hadrons within the finite-size hadronic matter in Refs. [12$14,22,25]$ may be a possible reason for being outside of our proposed band.

A similar kind of numerical band can be obtained from standard RTA expressions of bulk viscosity $(\zeta)$ as given in Eq. (2). These bands are shown by the cyan color in Fig. 5 .

After getting an approximate numerical band of transport coefficients of hadronic matter, now let us focus on absolute
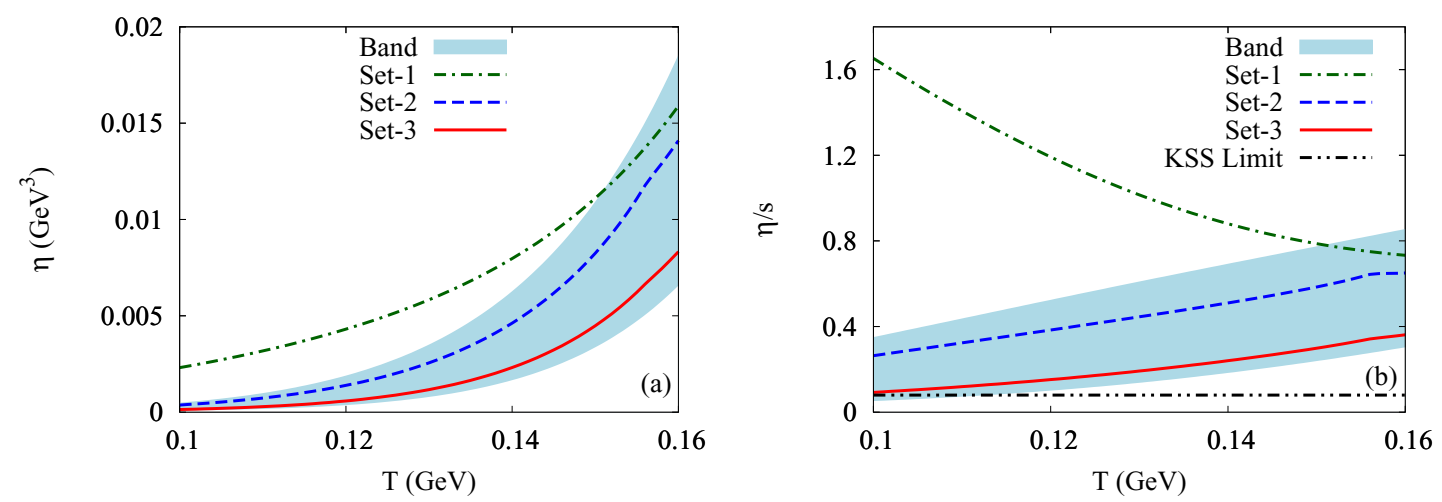

FIG. 4. (a) Shear viscosity ( $\eta$ ) and (b) shear viscosity to entropy density ratio $(\eta / s)$ as a function of temperature for Set-1, Set-2, and Set-3, as given in Table II. Approximate numerical bands of both are shown by cyan color and the dash-dot-dot line indicates the KSS limit for $\eta / s$. 

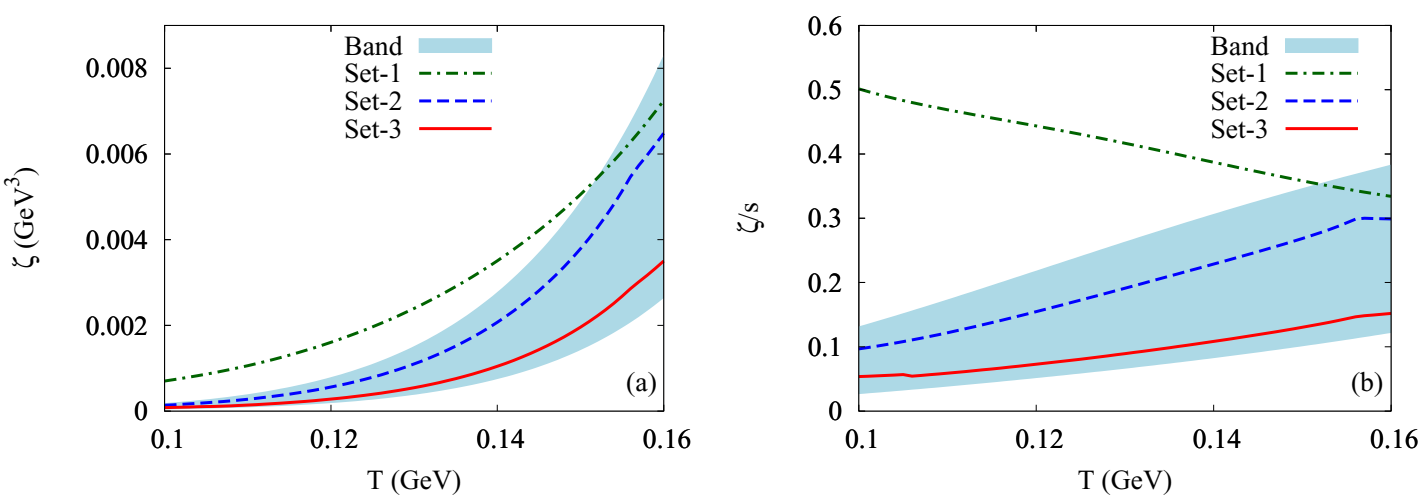

FIG. 5. The temperature dependence of (a) bulk viscosity $(\zeta)$ and (b) bulk viscosity to entropy density ratio $(\zeta / s)$ as a function of temperature for Set-1, Set-2, and Set-3 as given in Table II. Their approximate numerical band is also shown.

estimation. If we collect estimated values of transport coefficients for hadronic matter by earlier studies, then we will get a broad numerical band within which those estimations are located. In this regard, the present investigation provides a little narrow band and we are expecting that the values of transport coefficients for hadronic matter should be located within our proposed band, when one properly takes care about the finitesize dissipation phenomena. By taking an example, let us demonstrate how to consider the dissipation of hadrons within the finite-size hadronic matter and how it will help to reshape the values of transport coefficients within our proposed band. Let us calculate the relaxation times of NR particles from the experimentally available data of their scattering lengths. Here, we are consider scattering lengths $R_{a b}^{I}$ for different isospin $(I)$ states of $\pi \pi, \pi N, N N, K N$ interactions from Refs. [50,51] and $\pi K$ interaction from Refs. [53]. These input details are displayed in Table I. The reader can also find these values of scattering lengths collectively in Table 1 of Ref. [49] and Table 4 of Ref. [52]. Now using these values, we calculated isospin-average cross sections

$$
\sigma_{a b}=\sum_{I}(2 I+1) 4 \pi\left|R_{a b}^{I}\right|^{2} / \sum_{I}(2 I+1),
$$

TABLE I. Experimental values of scattering lengths of two-body elastic scattering of $\pi, N$, and $K$, taken from Refs. [50,51,53].

\begin{tabular}{llc}
\hline \hline$H H$ & \multicolumn{1}{c}{$a_{H H}^{I}$} & $\sigma_{H H}=\frac{\sum(2 I+1) 4 \pi\left|a_{H H}^{I}\right|^{2}}{\sum(2 I+1)}$ \\
\hline$\pi \pi$ & $a_{\pi \pi}^{I=0}=+0.37 \mathrm{fm}$ & \\
& $a_{\pi \pi}^{I=0}=-0.04 \mathrm{fm}$ & $\sigma_{\pi \pi}=17.3 \mathrm{mb}$ \\
$\pi N$ & $a_{\pi N}^{I=1 / 2}=+0.24 \mathrm{fm}$ & \\
& $a_{\pi N}^{I=3 / 2}=-0.14 \mathrm{fm}$ & $\sigma_{\pi N}=16.1 \mathrm{mb}$ \\
$N N$ & $a_{N N}^{I=0}=+20.1 \mathrm{fm}$ & \\
& $a_{N N}^{I=1}=-5.4 \mathrm{fm}$ & \\
$K N$ & $a_{K N}^{I=0}=-0.007 \mathrm{fm}$ & \\
& $a_{K N}^{I=1}=-0.225 \mathrm{fm}$ & \\
$K \pi$ & $a_{K \pi}^{I=1 / 2}=-0.22 \mathrm{fm}$ & \\
& $a_{K \pi}^{I=3 / 2}=-0.04 \mathrm{fm}$ & $\sigma_{K N}=4.7 \mathrm{mb}$ \\
\hline \hline
\end{tabular}

and then, we can calculated the relaxation time $\tau_{a}(a=\pi, K$ and $N$ ) from the the relation

$$
\frac{1}{\tau_{a}\left(\vec{k}_{a}\right)}=\sum_{b \in\{\pi, K, N\}} \int \frac{d^{3} \vec{k}_{b}}{(2 \pi)^{3}}\left[\sigma_{a b} v_{a b} n_{b}\right],
$$

where $n_{b}$ is the BE/FD distribution function of a meson/baryon;

$$
v_{a b}=\left(\frac{1}{2 \omega_{a} \omega_{b}}\right) \sqrt{\left\{s-\left(m_{a}+m_{b}\right)^{2}\right\}\left\{s-\left(m_{a}-m_{b}\right)^{2}\right\}}
$$

is the relative velocity with $\omega_{a, b}=\sqrt{\vec{k}_{a, b}^{2}+m_{a, b}^{2}}$; and $s=$ $\left(\omega_{a}+\omega_{b}\right)$. With the help of the relaxation time $\tau_{a}$ of $\pi, K$, and $N$, one can calculate their relaxation length $\lambda_{a}=$ $\vec{k}_{a} \tau_{a} / \omega_{a}$, as shown in Fig. 6. Here we see that relaxation lengths for $\pi$ and $K$ exceed the dimension of the fireball in the high momentum domain; although the nucleon relaxation length always remains lower than the dimension of the fireball in the entire momentum range. Now, let us use these entire momentum distributions of $\pi, K$, and $N$ in Eqs. (1) and (2) to get the NR contribution of $\eta$ and $\zeta$. Then after adding the contribution of the R component, we will get the total as shown by the green dashed dotted line in Figs. 4(a) and 5(a). Their dimensionless, normalized values, quantified as $\eta / s$ and $\zeta / s$, respectively, are shown by the green dashed dotted line in Figs. 4(b) and 5(b). All curves are going beyond the upper bound of our proposed numerical band. The reason is that we are considering high momentum $\pi$ and $K$, which are not at all participants in the dissipation process as their relaxation lengths exceed the system size. This can be well visualized from Fig. 6.

To resolve it, we first track numerically the upper momentum threshold or cutoff at different temperatures for $\pi$ and $K$, within which their relaxation lengths do not exceed the fireball dimension $(10 \mathrm{fm})$. From Fig. 6, one can visualize this fact graphically and then we plotted the the upper momentum thresholds $\vec{k}_{\text {th }}$ for $\pi$ and $\mathrm{K}$ as a function of temperature, which is shown in Fig. 7. Now when we put those $T$-dependent momentum thresholds as the upper limit in the integration of Eq. (1) and use the modified results of $\pi$ and $K$, the total values of $\eta, \eta / s, \zeta$, and $\zeta / s$ will politely remain within the numerical band. It is shown by the blue dashed lines in 

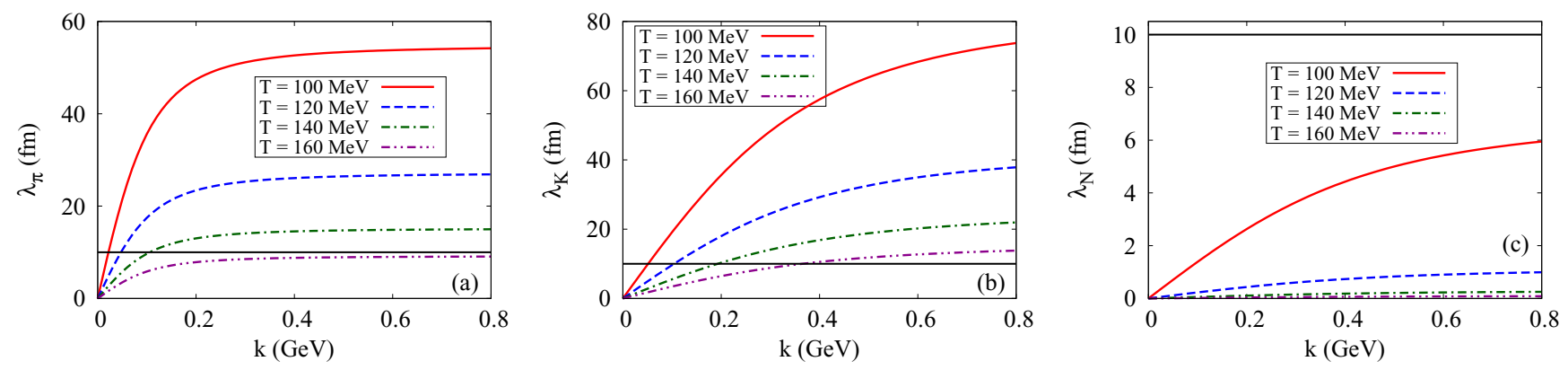

FIG. 6. Momentum distribution of relaxation lengths for (a) $\pi$, (b) $K$, and (c) $N$ at different temperatures.

Figs. 4 and 5. Hence, our investigation states that the values of transport coefficients for hadronic matter will be within our proposed numerical band, when one will properly impose the finite-size dissipation of the NR and R components during the calculation. We address this realistic or phenomenological issue, which should be considered for transport coefficients' calculations of hadronic matter, which is not infinite in size.

We may further extend our estimations by adopting the folding technique, described by Eq. (9). Putting $\eta, \zeta$ from Eqs. (1) and (2) in $\Phi$, we will get their modified results, as shown by the red solid lines in Figs. 4(a) and 5(a). We notice that the values of $\eta$ and $\zeta$ becomes lower due to the folding effect. For the convenience of the reader, Table II shows our different set of input choices, which we considered.

\section{SUMMARY AND DISCUSSION}

In summary, we pointed out a phenomenological issue of hadron resonance gas model, which should be seriously considered during the calculation of shear viscosity for RHIC or LHC matter and the facts are as follows. At first, on the basis of the dissipation process, we classified our HRG members into two categories-nonresonance members $(\pi, K$, and $N$ ) and resonance members (hadrons other than pseudoscalar meson nonet and baryon octet). Former members participate in dissipation via strong interaction scattering processes, where as the contribution from latter members is coming from their

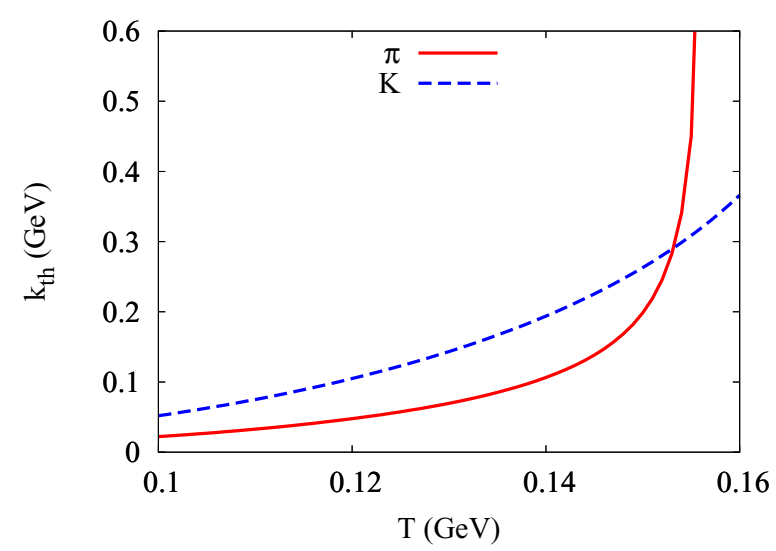

FIG. 7. Temperature dependence of upper momentum threshold $\vec{k}_{\mathrm{th}}$ for $\pi, K$. Beyond the threshold, their relaxation lengths exceed the fireball dimension. strong decay processes. We consider only strong interaction processes as other interaction (weak or electromagnetic) processes are meaningless for this scale of the (hadronic) system [15]. Beyond this normal filtering, we chose only those strong decays, whose mean life times are not exceeding the life time of the hadronic medium, which is roughly chosen as $10 \mathrm{fm}$. Now selecting those resonances and using their mean life times as relaxation times in the expression of shear viscosity, we get some nonzero value of $\eta$, which always has to be considered as a background value due to resonances in the HRG model. Taking Compton lengths $\left(1 / m_{\pi, K, N}\right)$ as minimum scattering lengths of NR particles $(\pi, K, N)$, we get a lower limit estimation for the NR component, which has to be added with the resonance component. We notice that the lower limit of total $\eta$, normalized by entropy density $s$ is greater than its quantum lower bound at the high-temperature range. Owing to this fact, we may conclude that $\eta / s$ in the HRG model never reaches its quantum lower bound near the transition temperature $T_{c}$ because of an unavoidable resonance contribution.

Similar to the lower limit, the upper limit of shear viscosity can be tuned by equating the relaxation length of NR particles to the dimension of the medium, produced in heavy ion experiments. The contribution from the resonance component is very definite or known since it is determined from the experimental values of mean life time of their strong decays, documented in PDG [54]. Hence, only adjustable quantities are relaxation lengths of NR particles, whose lower and upper possible values basically give a narrow numerical band in

TABLE II. Different set of inputs for transport coefficients $(\eta, \zeta)$ and entropy density (or other thermodynamical quantities like pressure, speed of sound).

\begin{tabular}{lcc}
\hline \hline & $\begin{array}{c}\text { Transport } \\
\text { coefficients }\end{array}$ & $\begin{array}{c}\text { Entropy } \\
\text { density }\end{array}$ \\
\hline Lower bound & NR $\left(\tau_{\text {low }}\right)$ & - \\
Upper bound & $+\mathrm{R}(\tau<10 \mathrm{fm})$ & \\
& $\mathrm{NR}(\tau=10 \mathrm{fm})$ & - \\
Set-1 & $+\mathrm{R}(\tau<10 \mathrm{fm})$ & $\mathrm{NR}+\mathrm{R}$ \\
Set-2 & $\mathrm{NR}+\mathrm{R}(\tau<10 \mathrm{fm})$ & - \\
& $\mathrm{NR}(\tau(\vec{k})<10 \mathrm{fm})$ & \\
Set-3 & $+\mathrm{R}(\tau<10 \mathrm{fm})$ & Set-1 with folding \\
\hline \hline
\end{tabular}


shear viscosity and other transport coefficients of hadronic matter.

Next, we take a particular example, where the absolute values of transport coefficients are obtained. Here, we estimate absolute values of relaxation lengths for nonresonance particles from their scattering length data. From the momentum distribution of their relaxation lengths, we find that the pion and kaon relaxation lengths exceed the fireball dimension beyond some upper values of momentum, which is again different for different temperature. Now when we take this temperature-dependent momentum cutoff as an upper limit of integration, then the values of the transport coefficients remain within our proposed numerical band. However, when we take the entire momentum distribution, those values do not remain within the band. Through this example, we want to emphasize the point - the values of transport coefficients for hadronic matter will remain within our proposed numerical band if we impose the idea of finite-size dissipation.

\section{ACKNOWLEDGMENTS}

Snigdha Ghosh acknowledges the Center for Nuclear Theory (CNT), Variable Energy Cyclotron Centre (VECC), funded by Department of Atomic Energy (DAE) and Indian Institute of Technology Gandhinagar, funded by Ministry of Human Resource Development (MHRD) for support. Sabyasachi Ghosh is partially supported by a University Grant Commission (UGC) Dr. D. S. Kothari Post Doctoral Fellowship (India) under Grant No. F.4-2/2006 (BSR)/PH/1516/0060. S.B. is supported from CSIR. We also thank Rajarshi Ray and Vinod Chandra for their useful suggestions.

\section{APPENDIX: HRG}

The momentum integral in Eq. (3) can be performed analytically and be expressed in terms of modified Bessel functions $K_{n}(x)$. Bellow we summarize the final forms of all the thermodynamic quantities:

$$
\begin{aligned}
& \ln \mathcal{Z}=\frac{V T^{3}}{2 \pi^{2}} \sum_{h \in\{\text { hadrons }\}} \sum_{n=1}^{\infty} g_{h} \frac{\left(a_{h}\right)^{n+1}}{n^{2}}\left(\frac{m_{h}}{T}\right)^{2} K_{2}\left(\frac{n m_{h}}{T}\right) \exp \left(\frac{n \mu_{h}}{T}\right), \\
& P=\frac{T^{4}}{2 \pi^{2}} \sum_{h \in\{\text { hadrons }\}} \sum_{n=1}^{\infty} g_{h} \frac{\left(a_{h}\right)^{n+1}}{n^{2}}\left(\frac{m_{h}}{T}\right)^{2} K_{2}\left(\frac{n m_{h}}{T}\right) \exp \left(\frac{n \mu_{h}}{T}\right), \\
& \varepsilon=\frac{T^{4}}{2 \pi^{2}} \sum_{h \in\{\text { hadrons }\}} \sum_{n=1}^{\infty} g_{h} \frac{\left(a_{h}\right)^{n+1}}{n^{2}}\left(\frac{m_{h}}{T}\right)^{2} \exp \left(\frac{n \mu_{h}}{T}\right)\left[\left\{1-\left(\frac{n \mu_{h}}{T}\right)\right\} K_{2}\left(\frac{n m_{h}}{T}\right)\right. \\
& \left.+\frac{1}{2}\left(\frac{n m_{h}}{T}\right)\left\{K_{1}\left(\frac{n m_{h}}{T}\right)+K_{3}\left(\frac{n m_{h}}{T}\right)\right\}\right] \\
& n_{k}=\frac{T^{3}}{2 \pi^{2}} \sum_{h \in\{\text { hadrons }\}} \sum_{n=1}^{\infty} g_{h} q_{h}^{k} \frac{\left(a_{h}\right)^{n+1}}{n}\left(\frac{m_{h}}{T}\right)^{2} K_{2}\left(\frac{n m_{h}}{T}\right) \exp \left(\frac{n \mu_{h}}{T}\right) ; k=B, Q, S, \ldots, \\
& \left(\frac{\partial P}{\partial T}\right)=\frac{T^{3}}{2 \pi^{2}} \sum_{h \in\{\text { hadrons }\}} \sum_{n=1}^{\infty} g_{h} \frac{\left(a_{h}\right)^{n+1}}{n^{2}}\left(\frac{m_{h}}{T}\right)^{2} \exp \left(\frac{n \mu_{h}}{T}\right)\left[\left\{2-\left(\frac{n \mu_{h}}{T}\right)\right\} K_{2}\left(\frac{n m_{h}}{T}\right)\right. \\
& \left.+\frac{1}{2}\left(\frac{n m_{h}}{T}\right)\left\{K_{1}\left(\frac{n m_{h}}{T}\right)+K_{3}\left(\frac{n m_{h}}{T}\right)\right\}\right] \\
& \left(\frac{\partial \varepsilon}{\partial T}\right)=\frac{T^{3}}{2 \pi^{2}} \sum_{h \in\{\text { hadrons }\}} \sum_{n=1}^{\infty} g_{h} \frac{\left(a_{h}\right)^{n+1}}{n^{2}}\left(\frac{m_{h}}{T}\right)^{2} \exp \left(\frac{n \mu_{h}}{T}\right)\left[\frac{1}{4}\left(\frac{n m_{h}}{T}\right)^{2}\left\{K_{0}\left(\frac{n m_{h}}{T}\right)+K_{4}\left(\frac{n m_{h}}{T}\right)\right\}\right. \\
& +\frac{1}{2}\left(\frac{n m_{h}}{T}\right)\left\{3-\left(\frac{n \mu_{h}}{T}\right)\right\}\left\{K_{1}\left(\frac{n m_{h}}{T}\right)+K_{3}\left(\frac{n m_{h}}{T}\right)\right\} \\
& \left.+\left\{2-2\left(\frac{n \mu_{h}}{T}\right)+\left(\frac{n \mu_{h}}{T}\right)^{2}+\frac{1}{2}\left(\frac{n m_{h}}{T}\right)^{2}\right\} K_{2}\left(\frac{n m_{h}}{T}\right)\right], \\
& \left(\frac{\partial P}{\partial \mu_{k}}\right)=n_{k} ; k=B, Q, S, \ldots, \\
& \left(\frac{\partial \varepsilon}{\partial \mu_{k}}\right)=\frac{T^{3}}{2 \pi^{2}} \sum_{h \in\{\text { hadrons }\}} \sum_{n=1}^{\infty} g_{h} q_{h}^{k} \frac{\left(a_{h}\right)^{n+1}}{n}\left(\frac{m_{h}}{T}\right)^{2} \exp \left(\frac{n \mu_{h}}{T}\right)\left[\frac{1}{2}\left(\frac{n m_{h}}{T}\right)\left\{K_{1}\left(\frac{n m_{h}}{T}\right)+K_{3}\left(\frac{n m_{h}}{T}\right)\right\}\right. \\
& \left.-\left(\frac{n \mu_{h}}{T}\right) K_{2}\left(\frac{n m_{h}}{T}\right)\right] ; k=B, Q, S, \ldots
\end{aligned}
$$


[1] P. K. Kovtun, D. T. Son, and A. O. Starinets, Phys. Rev. Lett. 94, 111601 (2005).

[2] J. Kinast, A. Turlapov, and J. E. Thomas, Phys. Rev. Lett. 94, 170404 (2005).

[3] M. Muller, J. Schmalian, and L. Fritz, Phys. Rev. Lett. 103, 025301 (2009).

[4] D. Mondal, D. Pandit, S. Mukhopadhyay, S. Pal, B. Dey, S. Bhattacharya, A. De, S. Bhattacharya, S. Bhattacharyya, P. Roy, K. Banerjee, and S. R. Banerjee, Phys. Rev. Lett. 118, 192501 (2017).

[5] M. Prakash, M. Prakash, R. Venugopalan, and G. Welke, Phys. Rep. 227, 321 (1993).

[6] K. Itakura, O. Morimatsu, and H. Otomo, Phys. Rev. D 77, 014014 (2008).

[7] A. Dobado and S. N. Santalla, Phys. Rev. D 65, 096011 (2002); A. Dobado and F. J. Llanes-Estrada, ibid. 69, 116004 (2004).

[8] D. Fernandez-Fraile and A. Gomez Nicola, Eur. Phys. J. C 62, 37 (2009).

[9] R. Lang, N. Kaiser, and W. Weise, Eur. Phys. J. A 48, 109 (2012).

[10] S. Mitra, S. Ghosh, and S. Sarkar, Phys. Rev. C 85, 064917 (2012).

[11] S. Ghosh, G. Krein, and S. Sarkar, Phys. Rev. C 89, 045201 (2014); S. Ghosh, ibid. 90, 025202 (2014); Braz. J. Phys. 45, 687 (2015).

[12] M. I. Gorenstein, M. Hauer, and O. N. Moroz, Phys. Rev. C 77, 024911 (2008).

[13] G. P. Kadam and H. Mishra, Phys. Rev. C 92, 035203 (2015).

[14] J. Noronha-Hostler, J. Noronha, and C. Greiner, Phys. Rev. Lett. 103, 172302 (2009).

[15] P. Chakraborty and J. I. Kapusta, Phys. Rev. C 83, 014906 (2011).

[16] C. Sasaki and K. Redlich, Nucl. Phys. A 832, 62 (2010).

[17] R. Marty, E. Bratkovskaya, W. Cassing, J. Aichelin, and H. Berrehrah, Phys. Rev. C 88, 045204 (2013).

[18] S. Ghosh, T. C. Peixoto, V. Roy, F. E. Serna, and G. Krein, Phys. Rev. C 93, 045205 (2016).

[19] P. Deb, G. P. Kadam, and H. Mishra, Phys. Rev. D 94, 094002 (2016).

[20] A. N. Tawfik, A. M. Diab, and M. T. Hussein, Int. J. Mod. Phys. A 31, 1650175 (2016).

[21] S. Ghosh, A. Lahiri, S. Majumder, R. Ray, and S. K. Ghosh, Phys. Rev. C 88, 068201 (2013).

[22] N. Demir and S. A. Bass, Phys. Rev. Lett. 102, 172302 (2009).

[23] A. Muronga, Phys. Rev. C 69, 044901 (2004).

[24] S. Plumari, A. Puglisi, F. Scardina, and V. Greco, Phys. Rev. C 86, 054902 (2012).

[25] S. Pal, Phys. Lett. B 684, 211 (2010).

[26] J.-B. Rose, J. M. Torres-Rincon, A. Schäfer, D. R. Oliinychenko, and H. Petersen, Phys. Rev. C 97, 055204 (2018).

[27] K. Paech and S. Pratt, Phys. Rev. C 74, 014901 (2006).
[28] P. Arnold, C. Dogan, and G. D. Moore, Phys. Rev. D 74, 085021 (2006).

[29] S. K. Das and Jan-e Alam, Phys. Rev. D 83, 114011 (2011).

[30] H. B. Meyer, Phys. Rev. Lett. 100, 162001 (2008).

[31] A. Dobado, F. J. Llane-Estrada, and J. Torres Rincon, Phys. Lett. B 702, 43 (2011).

[32] A. Dobado and J. M. Torres-Rincon, Phys. Rev. D 86, 074021 (2012).

[33] C. Sasaki and K. Redlich, Phys. Rev. C 79, 055207 (2009).

[34] X. Shi-Song, G. Pan-Pan, Z. Le, and H. De-Fu, Chin. Phys. C 38, 054101 (2014).

[35] D. Kharzeev and K. Tuchin, J. High Energy Phys. 09 (2008) 093.

[36] F. Karsch, D. Kharzeev, and K. Tuchin, Phys. Lett. B 663, 217 (2008).

[37] V. Chandra, Phys. Rev. D 86, 114008 (2012); 84, 094025 (2011).

[38] D. Fernandez-Fraile and A. Gomez Nicola, Phys. Rev. Lett. 102, 121601 (2009).

[39] S. Mitra and S. Sarkar, Phys. Rev. D 87, 094026 (2013); S. Mitra, U. Gangopadhyaya, and S. Sarkar, ibid. 91, 094012 (2015).

[40] S. Ghosh, S. Chatterjee, and B. Mohanty, Phys. Rev. C 94, 045208 (2016).

[41] G. Sarwar, S. Chatterjee, and J. Alam, J. Phys. G 44, 055101 (2017).

[42] G. P. Kadam and H. Mishra, Phys. Rev. C 93, 025205 (2016).

[43] K. Saha, S. Upadhaya, and S. Ghosh, Mod. Phys. Lett. A 32, 1750018 (2016).

[44] S. Gavin, Nucl. Phys. A 435, 826 (1985).

[45] S. Ghosh, Int. J. Mod. Phys. A 29, 1450054 (2014).

[46] P. Braun-Munzinger, K. Redlich, and J. Stachel, Quark Gluon Plasma 3, edited by R. C. Hwa and X. N. Wang (World Scientific, Singapore, 2004).

[47] S. Borsanyi, Z. Fodor, C. Hoelbling, S. D. Katz, S. Krieg, and K. K. Szabo, Phys. Lett. B 730, 99 (2014).

[48] A. Bazavov, T. Bhattacharya, C. DeTar, H.-T. Ding, S. Gottlieb, R. Gupta, P. Hegde, U. M. Heller, F. Karsch, E. Laermann, L. Levkova, S. Mukherjee, P. Petreczky, C. Schmidt, C. Schroeder, R. A. Soltz, W. Soeldner, R. Sugar, M. Wagner, and P. Vranas (HotQCD Collaboration), Phys. Rev. D 90, 094503 (2014).

[49] M. Fukugita, Y. Kuramashi, M. Okawa, H. Mino, and A. Ukawa, Phys. Rev. D 52, 3003 (1995).

[50] M. M. Nagels et al., Nucl. Phys. B 147, 189 (1979).

[51] O. Dumbrajs et al., Nucl. Phys. B 216, 277 (1983).

[52] N. T. Xiem and S. Shinmura, Prog. Theor. Exp. Phys. 2014, 023D04 (2014).

[53] P. Buttiker, S. Descotes-Genon, and B. Moussallam, Eur. Phys. J. C 33, 409 (2004).

[54] C. Patrignani et al. (Particle Data Group), Chin. Phys. C 40, 100001 (2016). 